PROCEEDINGS OF THE

AMERICAN MATHEMATICAL SOCIETY

Volume 135, Number 6, June 2007, Pages 1753-1762

S 0002-9939(07)08652-2

Article electronically published on January 31, 2007

\title{
SYMMETRY OF SOLUTIONS TO SEMILINEAR ELLIPTIC EQUATIONS VIA MORSE INDEX
}

\author{
FILOMENA PACELLA AND TOBIAS WETH
}

(Communicated by David S. Tartakoff)

\begin{abstract}
In this paper we prove symmetry results for solutions of semilinear elliptic equations in a ball or in an annulus in $\mathbb{R}^{N}, N \geq 2$, in the case where the nonlinearity has a convex first derivative. More precisely we prove that solutions having Morse index $j \leq N$ are foliated Schwarz symmetric, i.e. they are axially symmetric with respect to an axis passing through the origin and nonincreasing in the polar angle from this axis. From this we deduce, under some additional hypotheses on the nonlinearity, that the nodal set of sign changing solutions with Morse index $j \leq N$ intersects the boundary of the domain.
\end{abstract}

\section{INTRODUCTION}

In this paper we study symmetry properties of classical solutions to semilinear elliptic problems of the type

$$
\left\{\begin{aligned}
-\Delta u & =f(|x|, u) & & \text { in } B, \\
u & =0 & & \text { on } \partial B,
\end{aligned}\right.
$$

where $B$ is either a ball or an annulus centered at zero in $\mathbb{R}^{N}, N \geq 2$, and $f$ : $\bar{B} \times \mathbb{R} \rightarrow \mathbb{R}$ is (locally) a $C^{1, \alpha}$-function.

It is well known that a classical tool to study this question is the moving plane method which goes back to Alexandrov and Serrin [11] and was successfully used by Gidas-Ni-Nirenberg in the famous paper [9] to prove the radial symmetry of positive solutions to (1.1) when $B$ is a ball and $f$ has some monotonicity in the radial variable. However, if some of the hypotheses fail, as for example, if $u$ changes sign or $B$ is an annulus or $f$ does not have the right monotonicity, the moving plane method cannot be applied. Indeed, in each of these cases there are counterexamples to the radial symmetry of solutions. Nevertheless, for some nonlinearities, or for certain types of solutions, it is natural to expect that the solution inherits part of the symmetry of the domain. A first result in this direction has been obtained in 10] with a simple idea, based on the maximum principle, exploiting the Morse index of the solution. More precisely, in [10] it was proved that if $B$ is a ball or an annulus and $f(|x|, s)$ is strictly convex in the $s$-variable, then any solution $u$ to (1.1) with Morse index one is axially symmetric with respect to an axis passing through

Received by the editors September 30, 2005 and, in revised form, January 31, 2006.

2000 Mathematics Subject Classification. Primary 35J60.

The first author's research was supported by M.I.U.R., project 'Variational Methods and Nonlinear Differential Equations'. 
the origin and nonincreasing in the polar angle from this axis, i.e. only depends on $r=|x|$ and $\theta=\arccos \left(\frac{x}{|x|} \cdot p\right)$, for a certain unit vector $p$, and $u$ is nonincreasing in $\theta$. This kind of symmetry is often called foliated Schwarz symmetry.

Afterwards a similar result for minimizers of some variational problems was obtained in 13 using a completely different approach based on symmetrization techniques.

Though the result of [10] also applies to sign changing solutions of (1.1), there are cases when the results of [10] do not apply. Indeed, often sign changing solutions have Morse index greater than one, or the nonlinearity $f$, when considered on the whole real line, is not convex in the second variable. This is, for example, the case for the simple nonlinearity given by $f(s)=|s|^{p-1} s, p>1$. Moreover, also the results of [13] do not apply, because they essentially deal with cases where the minimizer is known to be a positive function. A first result, concerning variational problems where minimizers are sign changing functions, has been obtained recently in [3] where, using again symmetrization techniques, the authors extend the results of $[13]$.

In this paper we go back to the approach of [10] based on the maximum principle and Morse index information, and prove general symmetry results for solutions to (1.1) with 'high' Morse index, in the case where $f$ has its first derivative, with respect to the second variable, convex in the second variable. In the case of a power-like nonlinearity such as $f(s)=|s|^{p-1} s$ it means that we assume $p \geq 2$. From this we deduce the foliated Schwarz symmetry of solutions to (1.1) of Morse index less than or equal to $N$, exploiting some preliminary results obtained in Section 2. Our main result is the following.

Theorem 1.1. Suppose that $B$ is a ball in $\mathbb{R}^{N}, N \geq 2$, and $f(|x|, s)$ has a convex derivative $f^{\prime}(|x|, s)=\frac{\partial f}{\partial s}(|x|, s)$, for every $x \in B$. Then every solution of (1.1) with Morse index $j \leq N$ is foliated Schwarz symmetric.

To our knowledge this is the first general result in this direction, i.e. in showing that solutions with any Morse index must be foliated Schwarz symmetric if the dimension is sufficiently large. An indication that also solutions with high Morse index should have some symmetry is contained in 7, where the symmetry of a particular type of solutions which blow up in several points, in an annulus, is analyzed (see also [6]).

Let us remark that indeed there are only two possibilities under the assumptions of Theorem 1.1. Either the solution is radially symmetric or it is strictly monotone in the polar angle. This follows easily from the argument in Proposition 2.3 below where we study the sign of angular derivatives of the solution. The first alternative occurs for solutions having Morse index zero, as pointed out in Remark 3.3 below.

We also show an interesting consequence of Theorem 1.1 concerning the geometrical properties of the nodal set of a sign changing solution of (1.1) which relies on some results of 1 .

Theorem 1.2. Suppose that $B$ is a ball in $\mathbb{R}^{N}, N \geq 2$, and $f=f(s)$ does not depend on $x$. Suppose furthermore that $f^{\prime}$ is convex. Then the nodal set of any sign changing solutions of (1.1) with Morse index less than or equal to $N$ intersects the boundary of $B$.

Note that the assumptions of Theorem 1.2 are satisfied for the power type nonlinearity $f(s)=|s|^{p-1} s, p \geq 2$. In particular, under these assumptions, from 
Theorem 1.2 it follows that every sign changing solution $u$ of (1.1) with Morse index less than or equal to $N$ is nonradial. This was already proved in [1] by showing that any sign changing radial solution has Morse index greater than or equal to $N+1$.

The outline of the paper is the following. In Section 2 we recall the necessary definitions and prove preliminary results on foliated Schwarz symmetry. In Section 3 we prove Theorem 1.1 and Theorem 1.2 .

\section{Preliminary Results}

Let $u \in H_{0}^{1}(B)$ be a solution of (1.1). By elliptic regularity theory, $u \in C^{3, \alpha}(\bar{B})$ for some $\alpha>0$. In particular $V_{u}(x):=f^{\prime}(|x|, u(x))=\frac{\partial f}{\partial u}(|x|, u(x))$ is continuous on $\bar{B}$. Hence we may consider the linearized operator

$$
L: H^{2}(B) \cap H_{0}^{1}(B) \subset L^{2}(B) \rightarrow L^{2}(B), \quad L v=-\Delta v-V_{u} v .
$$

The operator $L$ is selfadjoint, and its spectrum consists of a sequence of eigenvalues $\lambda_{1}<\lambda_{2} \leq \lambda_{3} \leq \cdots \leq \lambda_{k} \rightarrow \infty$. We recall that the Morse index of $u$ is the number of negative eigenvalues of $L$. Now we recall the definition of foliated Schwarz symmetry.

Definition 2.1. We say that a function $v \in \mathcal{C}(\bar{B})$ is foliated Schwarz symmetric if there is a unit vector $p \in \mathbb{R}^{N},|p|=1$ such that $v(x)$ only depends on $r=|x|$ and $\theta:=\arccos \left(\frac{x}{|x|} \cdot p\right)$, and $u$ is nonincreasing in $\theta$.

Let $S$ be the unit sphere in $\mathbb{R}^{N}, S=\left\{x \in \mathbb{R}^{N}:|x|=1\right\}$. For a unit vector $e \in S$ we consider the hyperplane $H(e)=\left\{x \in \mathbb{R}^{N}: x \cdot e=0\right\}$ and the open half domain $B(e)=\{x \in B: x \cdot e>0\}$. We write $\sigma_{e}: B \rightarrow B$ for the reflection with respect to $H(e)$, that is, $\sigma_{e}(x)=x-2(x \cdot e) e$ for every $x \in B$. Note that

$$
H(-e)=H(e) \quad \text { and } \quad B(-e)=\sigma_{e}(B(e))=-B(e) \quad \text { for every } \quad e \in S .
$$

We have

Lemma 2.2. Let $u \in H_{0}^{1}(B) \cap \mathcal{C}(\bar{B})$. Assume that for every unit vector $e \in S$ we either have $u(x) \geq u\left(\sigma_{e}(x)\right)$ for all $x \in B(e)$ or $u(x) \leq u\left(\sigma_{e}(x)\right)$ for all $x \in B(e)$. Then $u$ is foliated Schwarz symmetric.

This lemma can be found for instance in [5, Lemma 4.3] for the case of an annulus, but the proof carries over to the case of a ball. It is also implicitly contained in [3, Section 2]. We now give another surprisingly simple criterion for the foliated Schwarz symmetry. For this we denote by $\lambda_{k}\left(e, V_{u}\right)$ the eigenvalues of the operator $-\Delta-V_{u}(x)$ in the half domain $B(e)$, with homogeneous Dirichlet boundary conditions.

Proposition 2.3. Let $u$ be a solution of (1.1), and suppose that there exists $e \in$ $S$ such that $u$ is symmetric with respect to the hyperplane $H(e)$, and such that $\lambda_{1}\left(e, V_{u}\right) \geq 0$. Then $u$ is foliated Schwarz symmetric.

Proof. After a rotation, we may assume that $e=e_{2}=(0,1, \ldots, 0)$, hence $H(e)=$ $\left\{x \in \mathbb{R}^{N}: x_{2}=0\right\}$. We want to apply Lemma 2.2, so we consider an arbitrary unit vector $e^{\prime} \in S$ different from $\pm e$. After another orthogonal transformation which leaves $e_{2}$ and $H\left(e_{2}\right)$ invariant, we may assume that $e^{\prime}=\left(\cos \theta_{0}, \sin \theta_{0}, 0, \ldots, 0\right)$ for some $\theta_{0} \in\left(-\frac{\pi}{2}, \frac{\pi}{2}\right)$. Now we choose new coordinates, replacing $x_{1}, x_{2}$ by polar coordinates $r, \theta$ with $x_{1}=r \cos \theta, x_{2}=r \sin \theta$, and leaving $\tilde{x}:=\left(x_{3}, \ldots, x_{N}\right)$ 
unchanged. In these new (cylinder) coordinates, the Laplacian of a function $u=$ $u(r \cos \theta, r \sin \theta, \tilde{x})$ is given by

$$
\Delta u=\frac{\partial^{2} u}{\partial r^{2}}+\frac{1}{r} \frac{\partial u}{\partial r}+\frac{1}{r^{2}} \frac{\partial^{2} u}{\partial \theta^{2}}+\sum_{i=3}^{N} \frac{\partial^{2} u}{\partial x_{i}^{2}} .
$$

In the following, we write $u_{\theta}$ for $\frac{\partial u}{\partial \theta}$. Differentiating the equation $-\Delta u=f(|x|, u)$ with respect to $\theta$, we obtain $-\Delta u_{\theta}=f^{\prime}(|x|, u) u_{\theta}=V_{u}(x) u_{\theta}$ in $B$. Moreover, $u_{\theta}=0$ on $\partial B$, since $u$ satisfies zero Dirichlet boundary conditions on $\partial B$. Hence $u_{\theta}$ is an eigenfunction of $L$ corresponding to the eigenvalue 0 . Moreover, since by assumption $u$ is symmetric with respect to $H\left(e_{2}\right)$, we have

$$
u(r \cos (-\theta), r \sin (-\theta), \tilde{x})=u(r \cos \theta,-r \sin \theta, \tilde{x})=u(r \cos \theta, r \sin \theta, \tilde{x})
$$

and therefore $u_{\theta}(r \cos (-\theta), r \sin (-\theta), \tilde{x})=-u_{\theta}(r \cos \theta, r \sin \theta, \tilde{x})$. Hence $u_{\theta}$ is antisymmetric with respect to the reflection at $H\left(e_{2}\right)$. We note that $u_{\theta}$ does not change sign in $B\left(e_{2}\right)$. Indeed, if $u_{\theta}$ changed sign in $B\left(e_{2}\right)$, then the restriction of $u_{\theta}$ to $B\left(e_{2}\right)$ would be a sign changing Dirichlet eigenfunction, corresponding to the zero eigenvalue, of the operator $-\Delta-V_{u}(x)$ on $B\left(e_{2}\right)$. This however contradicts the assumption $\lambda_{1}\left(e_{2}, V_{u}\right) \geq 0$. To proceed, we need to write the reflection $\sigma_{e^{\prime}}$ at the hyperplane $H\left(e^{\prime}\right)$ in the new coordinates. An easy calculation shows

$$
\sigma_{e^{\prime}}(r \cos \theta, r \sin \theta, \tilde{x})=\left(r \cos \left(2 \theta_{0}-\theta+\pi\right), r \sin \left(2 \theta_{0}-\theta+\pi\right), \tilde{x}\right) .
$$

We now distinguish two cases.

Case 1: $u_{\theta} \geq 0$ on $B\left(e_{2}\right)$ and $u_{\theta} \leq 0$ on $B\left(-e_{2}\right)$. Then we claim

$$
u \leq u \circ \sigma_{e^{\prime}} \quad \text { on } B\left(e^{\prime}\right) \text {. }
$$

Let $x=(r \cos \theta, r \sin \theta, \tilde{x}) \in B\left(e^{\prime}\right)$. Hence $\left|\theta-\theta_{0}\right|<\frac{\pi}{2}$. We first suppose $x \in B\left(e_{2}\right)$, which means that $\theta \in(0, \pi)$. If $\theta \geq 2 \theta_{0}$, then $0<\theta<2 \theta_{0}-\theta+\pi \leq \pi$, and therefore

$$
u(r \cos \theta, r \sin \theta, \tilde{x}) \leq u\left(r \cos \left(2 \theta_{0}-\theta+\pi\right), r \sin \left(2 \theta_{0}-\theta+\pi\right), \tilde{x}\right)=u\left(\sigma_{e^{\prime}}(x)\right) .
$$

On the other hand, if $\theta<2 \theta_{0}$, then $0<\theta<\pi-\left(2 \theta_{0}-\theta\right)<\pi$, and hence by (2.2)

$$
\begin{aligned}
u(r \cos \theta, r \sin \theta, \tilde{x}) & \leq u\left(r \cos \left(\pi-\left(2 \theta_{0}-\theta\right)\right), r \sin \left(\pi-\left(2 \theta_{0}-\theta\right)\right), \tilde{x}\right) \\
& =u\left(r \cos \left(2 \theta_{0}-\theta-\pi\right), r \sin \left(2 \theta_{0}-\theta-\pi\right), \tilde{x}\right) \\
& =u\left(r \cos \left(2 \theta_{0}-\theta+\pi\right), r \sin \left(2 \theta_{0}-\theta+\pi\right), \tilde{x}\right) \\
& =u\left(\sigma_{e^{\prime}}(x)\right) .
\end{aligned}
$$

Next we suppose $x \in B\left(-e_{2}\right)$, which means that $\theta \in(-\pi, 0)$. If $\theta \leq 2 \theta_{0}$, then $0>\theta>2 \theta_{0}-\theta-\pi \geq-\pi$, and therefore

$$
\begin{aligned}
u(r \cos \theta, r \sin \theta, \tilde{x}) & \leq u\left(r \cos \left(2 \theta_{0}-\theta-\pi\right), r \sin \left(2 \theta_{0}-\theta-\pi\right), \tilde{x}\right) \\
& =u\left(r \cos \left(2 \theta_{0}-\theta+\pi\right), r \sin \left(2 \theta_{0}-\theta+\pi\right), \tilde{x}\right) \\
& =u\left(\sigma_{e^{\prime}}(x)\right) .
\end{aligned}
$$

On the other hand, if $\theta>2 \theta_{0}$, then $0>\theta>-\pi-\left(2 \theta_{0}-\theta\right)>-\pi$, and hence by (2.2)

$$
\begin{aligned}
u(r \cos \theta, r \sin \theta, \tilde{x}) & \leq u\left(r \cos \left(-\pi-\left(2 \theta_{0}-\theta\right)\right), r \sin \left(-\pi-\left(2 \theta_{0}-\theta\right)\right), \tilde{x}\right) \\
& =u\left(r \cos \left(2 \theta_{0}-\theta+\pi\right), r \sin \left(2 \theta_{0}-\theta+\pi\right), \tilde{x}\right) \\
& =u\left(\sigma_{e^{\prime}}(x)\right) .
\end{aligned}
$$

So we have proved (2.3) in all circumstances. 
Case 2: $u_{\theta} \leq 0$ on $B\left(e_{2}\right)$ and $u_{\theta} \geq 0$ on $B\left(-e_{2}\right)$. Then a similar argument as in Case 1 shows that $u \geq u \circ \sigma_{e^{\prime}}$ on $B\left(e^{\prime}\right)$.

Thus Lemma 2.2 applies and yields that $u$ is foliated Schwarz symmetric.

\section{Proof of Theorems 1.1 and 1.2}

In this section we will use the same notations as in Section 2, in particular we denote by $e$ a direction in $\mathbb{R}^{N}$, i.e., a unit vector. Moreover, if $u$ is a solution of (1.1), for any direction $e \in S$ we denote by $V_{e s}$ the even part of the potential $V_{u}(x)=f^{\prime}(|x|, u(x))$ relative to the reflection with respect to the hyperplane $H(e)$, i.e., we define

$$
V_{e s}(x)=\frac{1}{2}\left[f^{\prime}(|x|, u(x))+f^{\prime}\left(|x|, u\left(\sigma_{e}(x)\right)\right)\right] .
$$

Then, we denote by $\lambda_{k}\left(e, V_{e s}\right)$ the eigenvalues of the operator $-\Delta-V_{e s}(x)$ in the half domain $B(e)$, with homogeneous Dirichlet boundary conditions. The following result gives a sufficient condition for the existence of a symmetry hyperplane for a solution of (1.1).

Proposition 3.1. Suppose that $f^{\prime}(|x|, s)$ is convex in $s$, and let $u$ be a solution of (1.1), and $e \in S$ a direction such that $\lambda_{1}\left(e, V_{e s}\right) \geq 0$. Then

(i) if either $\lambda_{1}\left(e, V_{e s}\right)>0$ or $f^{\prime}(|x|, s)$ is strictly convex in $s$, then $u$ is symmetric with respect to the hyperplane $H(e)$. Thus $\lambda_{1}\left(e, V_{u}\right)=\lambda_{1}\left(e, V_{e s}\right) \geq 0$.

(ii) there exists a possibly different direction $e^{\prime} \in S$ such that $u$ is symmetric with respect to the hyperplane $H\left(e^{\prime}\right)$, and $\lambda_{1}\left(e^{\prime}, V_{u}\right) \geq 0$.

Combining this with Proposition 2.3, we immediately obtain:

Corollary 3.2. Under the assumptions of Proposition 3.1, the solution u is foliated Schwarz symmetric.

Proof of Proposition 3.1. Let us denote by $w_{e}$ the difference between $u$ and its reflection with respect to the hyperplane $H(e)$, i.e., $w_{e}(x)=u(x)-u\left(\sigma_{e}(x)\right)$. It is easy to see that $w_{e}$ solves the linear problem

$$
\left\{\begin{aligned}
-\Delta w_{e}-V_{e}(x) w_{e}=0 & & \text { in } B(e), \\
w_{e}=0 & & \text { on } \partial B(e),
\end{aligned}\right.
$$

where

$$
V_{e}(x)=\int_{0}^{1} f^{\prime}\left(|x|, t u(x)+(1-t) u\left(\sigma_{e}(x)\right)\right) d t, \quad x \in B(e) .
$$

Since $f^{\prime}$ is convex in the second variable, we have

$$
\begin{aligned}
V_{e}(x) & \leq \int_{0}^{1}\left[t f^{\prime}(|x|, u(x))+(1-t) f^{\prime}\left(|x|, u\left(\sigma_{e}(x)\right)\right)\right] d t \\
& =\frac{1}{2}\left[f^{\prime}(|x|, u(x))+f^{\prime}\left(|x|, u\left(\sigma_{e}(x)\right)\right)\right]=V_{e s}(x)
\end{aligned}
$$

for $x \in B$. Here the strict inequality holds if $f^{\prime}$ is strictly convex and $u(x) \neq$ $u\left(\sigma_{e}(x)\right)$. Hence, denoting by $\lambda_{k}\left(e, V_{e}\right)$ the eigenvalues of the linear operator $-\Delta-$ $V_{e}(x)$ in $B(e)$ with homogeneous Dirichlet boundary conditions, we have by (3.4) that $\lambda_{k}\left(e, V_{e}\right) \geq \lambda_{k}\left(e, V_{e s}\right)$. In particular, $\lambda_{1}\left(e, V_{e}\right) \geq \lambda_{1}\left(e, V_{e s}\right) \geq 0$ by hypothesis. If $\lambda_{1}\left(e, V_{e}\right)>0$, then $w_{e} \equiv 0$ because it satisfies (3.2). Thus we get the symmetry of $u$ with respect to the hyperplane $H(e)$ in this case. If $\lambda_{1}\left(e, V_{e}\right)=0$, then $V_{e} \equiv V_{e s}$ 
in $B$ by the strict monotonicity of eigenvalues with respect to the potential, see e.g. [8. In the case where $f^{\prime}$ is strictly convex this implies that $u(x) \equiv u\left(\sigma_{e}(x)\right)$ for every $x \in B$, so that again we get the symmetry of $u$ with respect to the hyperplane $H(e)$.

It remains to consider the case where $f^{\prime}$ is only convex and $\lambda_{1}\left(e, V_{e}\right)=0$. In this case either $w_{e} \equiv 0$ or $w_{e}$ does not change sign in $B(e)$, and, by the strong maximum principle, $w_{e}>0$ or $w_{e}<0$ in $B(e)$. Without loss of generality, we assume that $e=(0,0, \ldots, 0,1)$ and $w_{e}>0$ in $B(e)$. Then we start rotating the plane $H(e)$ with this condition. Hence we set $e_{\theta}=(\sin \theta, 0, \ldots, 0, \cos \theta)$ for $\theta \geq 0$, so that $e_{0}=e$. For abbreviation we also put

$$
B_{\theta}:=B\left(e_{\theta}\right)=\left\{x \in B: x_{1} \sin \theta+x_{N} \cos \theta>0\right\} \quad \text { and } \quad w_{\theta}:=w_{e_{\theta}} .
$$

Note that, if $w_{\theta}>0$ in $B_{\theta}$ for some $\theta \geq 0$, then $\lambda_{1}\left(e_{\theta}, V_{e_{\theta}}\right)=0$ by (3.2). We define

$$
\tilde{\theta}=\sup \left\{\theta \in[0, \pi): w_{\theta}>0 \quad \text { in } B_{\theta}\right\} .
$$

Then, by continuity,

$$
\lambda_{1}\left(e_{\tilde{\theta}}, V_{e_{\tilde{\theta}}}\right)=0,
$$

and $w_{\tilde{\theta}} \geq 0$ in $B_{\tilde{\theta}}$. This in particular implies $\tilde{\theta}<\pi$, since $w_{\pi}=-w_{0}<0$ in $B_{\pi}=-B_{0}$. We would like to prove that $w_{\tilde{\theta}} \equiv 0$. Arguing by contradiction we assume that $w_{\tilde{\theta}} \not \equiv 0$, so that, by the strong maximum principle, $w_{\tilde{\theta}}>0$ in $B_{\tilde{\theta}}$. Now let $K \subset B_{\tilde{\theta}}$ be a compact set such that $B_{\tilde{\theta}} \backslash K$ has small measure to allow the strong maximum principle to hold for the operator $-\Delta-V_{e_{\tilde{\theta}+\varepsilon}}(x)$ in the set $B_{\tilde{\theta}+\varepsilon} \backslash K$ for sufficiently small $\varepsilon \geq 0$ (cf. e.g. [4, Prop. 1.1]). Since $w_{\tilde{\theta}}>\eta>0$ in $K$ for some $\eta>0$, we have

$$
w_{\tilde{\theta}+\varepsilon}>\frac{\eta}{2}>0 \quad \text { in } K \subset B_{\tilde{\theta}+\varepsilon}
$$

for sufficiently small $\varepsilon>0$, while

$$
w_{\tilde{\theta}+\varepsilon}>0 \quad \text { in } B_{\tilde{\theta}+\varepsilon} \backslash K
$$

by the strong maximum principle. Therefore $w_{\tilde{\theta}+\varepsilon}>0$ in $B_{\tilde{\theta}+\varepsilon}$, contradicting the definition of $\tilde{\theta}$. Hence $w_{\tilde{\theta}}$ must be identically zero, and so we have $u(x)=$ $u\left(\sigma_{e_{\tilde{\theta}}}(x)\right)$ for all $x \in B_{\theta}$. Finally, by (3.5) we have $\lambda_{1}\left(e_{\tilde{\theta}}, V_{u}\right)=\lambda_{1}\left(e_{\tilde{\theta}}, V_{e_{\tilde{\theta}}}\right)=0$, as claimed.

Remark 3.3. By Proposition 3.1 it follows that any solution of (1.1) of Morse index zero is radial if $f^{\prime}$ is convex in the second variable. Indeed, in this case the first eigenvalue $\lambda_{1}$ of the linearized operator $-\Delta-V_{u}(x)$ in $B$ is nonnegative. Moreover it is easy to see that $\lambda_{1}\left(B, V_{e s}\right) \geq \lambda_{1} \geq 0$, for any unit vector $e \in S$, denoting by $\lambda_{1}\left(B, V_{e s}\right)$ the first Dirichlet eigenvalue of the operator $-\Delta-V_{e s}$ in the whole domain $B$. Hence $\lambda_{1}\left(e, V_{e s}\right)>0$ for any direction $e$, which yields, by Proposition 3.1, the symmetry of the solution with respect to any hyperplane passing through the origin. 
Before passing to the proof of Theorem 1.1, let us fix some further notations. For a solution $u$ of (1.1) and functions $v, w \in H_{0}^{1}(B)$ we put

$$
\begin{aligned}
\langle v, w\rangle & =\int_{B} v(x) w(x) d x, \\
Q_{u}(v, w) & =\int_{B} \nabla v(x) \nabla w(x) d x-\int_{B} V_{u}(x) v(x) w(x) d x, \\
Q_{e s}(v, w) & =\int_{B} \nabla v(x) \nabla w(x) d x-\int_{B} V_{e s}(x) v(x) w(x) d x .
\end{aligned}
$$

Proof of Theorem 1.1. Let $u$ be a solution of (1.1) with Morse index $m(u)=j \leq N$. Thus, for the Dirichlet eigenvalues $\lambda_{k}$ of the linearized operator $L=-\Delta-V_{u}(x)$ in $B$ we have

$$
\lambda_{1}<0, \ldots, \lambda_{j}<0 \quad \text { and } \quad \lambda_{j+1} \geq 0 .
$$

We first assume that $j \leq N-1$ and show how to obtain the foliated Schwarz symmetry of $u$, applying Corollary 3.2 in a simple way. For any direction $e \in S$, let us denote by $g_{e} \in H_{0}^{1}(B)$ the odd extension in $B$ of the positive $L^{2}$-normalized eigenfunction of the operator $-\Delta-V_{e s}(x)$ in the half domain $B(e)$ corresponding to $\lambda_{1}\left(e, V_{e s}\right)$. It is easy to see that $g_{e}$ depends continuously on $e$ in the $L^{2}$-norm. Moreover, $g_{-e}=-g_{e}$ for every $e \in S$. Now we let $\varphi_{1}, \varphi_{2}, \ldots, \varphi_{j} \in H_{0}^{1}(B)$ denote $L^{2}$-orthonormal eigenfunctions of $L$ corresponding to the eigenvalues $\lambda_{1}, \ldots, \lambda_{j}$. It is well known that

$$
\inf _{\substack{v \in H_{0}^{1}(B) \backslash\{0\} \\\left\langle v, \varphi_{1}\right\rangle=\ldots=\left\langle v, \varphi_{j}\right\rangle=0}} \frac{Q_{u}(v, v)}{\langle v, v\rangle}=\lambda_{j+1} \geq 0 .
$$

We consider the map

$$
h: S \rightarrow \mathbb{R}^{j}, \quad h(e)=\left[\left\langle g_{e}, \varphi_{1}\right\rangle, \ldots,\left\langle g_{e}, \varphi_{j}\right\rangle\right] .
$$

Since $h$ is an odd and continuous map defined on the unit sphere $S \subset \mathbb{R}^{N}$ and $j \leq N-1, h$ must have a zero by the Borsuk-Ulam Theorem. This means that there is a direction $e \in S$ such that $g_{e}$ is $L^{2}$ orthogonal to all eigenfunctions $\varphi_{1}, \ldots, \varphi_{j}$. Thus $Q_{u}\left(g_{e}, g_{e}\right) \geq 0$ by (3.7). But, since $g_{e}$ is an odd function,

$$
Q_{u}\left(g_{e}, g_{e}\right)=Q_{e s}\left(g_{e}, g_{e}\right)=2 \lambda_{1}\left(e, V_{e s}\right),
$$

which yields that $\lambda_{1}\left(e, V_{e s}\right) \geq 0$. Having obtained a direction $e$ for which $\lambda_{1}\left(e, V_{e s}\right)$ is nonnegative, Corollary 3.2 applies and yields the foliated Schwarz symmetry of $u$.

Now we turn to the more difficult case where the Morse index $m(u)$ is equal to $N$. The main difficulty in this case is the fact that the map $h$, considered in (3.8), now goes from the $N$-1-dimensional sphere $S$ into $\mathbb{R}^{N}$, so that the Borsuk-Ulam Theorem does not apply. We therefore use a different and less direct argument to find a symmetry hyperplane $H\left(e^{\prime}\right)$ for $u$ with $\lambda_{1}\left(e^{\prime}, V_{u}\right) \geq 0$, so that Proposition 2.3 can be applied. Let $S_{*} \subset S$ be defined by

$$
S_{*}=\left\{e \in S: w_{e} \equiv 0 \text { in } B \text { and } \lambda_{1}\left(e, V_{u}\right)<0\right\} \text {, }
$$

where again $w_{e}$ is given by $w_{e}(x)=u(x)-u\left(\sigma_{e}(x)\right)$. Then $S_{*}$ is a symmetric set. If $S_{*} \neq \varnothing$ we let $k$ be the largest integer such that there exist $k$ orthogonal directions $e_{1}, \ldots, e_{k} \in S_{*}$. We then observe that $k \in\{1, \ldots, N-1\}$. Indeed, if we denote by $\mathcal{V}_{0}$ the closed subspace of $L^{2}(B)$ consisting of the functions which are symmetric 
with respect to the hyperplanes $H\left(e_{1}\right), \ldots, H\left(e_{k}\right)$, we have that $u \in \mathcal{V}_{0}$ (by the very definition of $S_{*}$ ) and also $\varphi_{1} \in \mathcal{V}_{0}$, where $\varphi_{1}$ denotes the unique positive $L^{2}$ normalized Dirichlet eigenfunction of the linearized operator $L=-\Delta-V_{u}(x)$ on $B$ relative to the first eigenvalue $\lambda_{1}$. Now we consider for $l=1, \ldots, k$ the functions $g_{l} \in H_{0}^{1}(B)$ defined as the odd extensions in $B$ of the unique positive $L^{2}$-normalized eigenfunction of the operator $-\Delta-V_{u}(x)$ in the half domain $B\left(e_{l}\right)$ corresponding to $\lambda_{1}\left(e_{l}, V_{u}\right)<0$. Then, since $\mathrm{u}$ is symmetric with respect to $H\left(e_{l}\right), g_{l}$ is an eigenfunction of the operator $-\Delta-V_{u}(x)$ in the whole ball $B$ corresponding to a certain eigenvalue $\lambda_{m}\left(=\lambda_{1}\left(e_{l}, V_{u}\right)<0\right), m \geq 2$. Since each $g_{l}$ is odd with respect to the reflection at $H\left(e_{l}\right)$ and even with respect to the reflection at $H\left(e_{m}\right)$ for $m \neq l$, we have, letting $l$ vary from 1 to $k$, a set $\left\{g_{1}, \ldots, g_{k}\right\}$ of $k$ orthogonal eigenfunctions of the operator $-\Delta-V_{u}(x)$, all corresponding to negative eigenvalues. Since the Morse index of $u$ is equal to $N$ and the first eigenfunction $\varphi_{1}$ is also orthogonal to each $g_{l}$, we have that $k \leq N-1$, as we claimed. The same argument shows that if we denote by $L_{0}$ the selfadjoint operator which is the restriction of the operator $L$ to the symmetric space $\mathcal{V}_{0}$, i.e.

$$
L_{0}: H_{2}(B) \cap H_{0}^{1}(B) \cap \mathcal{V}_{0} \rightarrow \mathcal{V}_{0}, \quad L_{0} v=-\Delta v-V_{u}(x) v,
$$

and we denote by $\mu_{0}$ the number of the negative eigenvalues of $L_{0}$ (counted with multiplicity), we have

$$
1 \leq \mu_{0} \leq N-k \text {. }
$$

Note that the inequality on the left just follows from the fact that $\varphi_{1} \in \mathcal{V}_{0}$ is an eigenfunction of $L$ relative to a negative eigenvalue. Now we first assume that $S_{*} \neq \varnothing$ and consider the $N-k-1$-dimensional sphere $S^{*}$ defined by $S^{*}=S \cap$ $H\left(e_{1}\right) \cap \cdots \cap H\left(e_{k}\right)$. By the maximality of $k$, we have $S^{*} \cap S_{*}=\varnothing$. We would like to prove that there exists a direction $e \in S^{*}$ such that

$$
w_{e}(x) \geq 0 \quad \text { for every } \quad x \in B(e) .
$$

We argue by contradiction and suppose that $w_{e}$ changes sign in $B(e)$ for every $e \in S^{*}$. We consider the functions $w_{e}^{1}=w_{e}^{+} \chi_{B(e)}-w_{e}^{-} \chi_{B(-e)}$ and $w_{e}^{2}=-w_{e}^{-} \chi_{B(e)}+$ $w_{e}^{+} \chi_{B(-e)}$, where $w^{+}=\max \{w, 0\}, w^{-}=\min \{w, 0\}$ and $\chi_{\Omega}$ denotes the characteristic function of a set $\Omega$. Since $u \in \mathcal{V}_{0}$, we find that $w_{e}^{1}, w_{e}^{2} \in \mathcal{V}_{0} \cap H_{0}^{1}(B) \backslash\{0\}$, and both functions are nonnegative and symmetric with respect to $\sigma_{e}$. Moreover,

$$
w_{-e}^{1}=w_{e}^{2} \quad \text { and } \quad w_{-e}^{2}=w_{e}^{1} \quad \text { for every } e \in S^{*} .
$$

Recalling the definition of $V_{e}$ in (3.3), since $w_{e}$ satisfies the linear equation $-\Delta w_{e}-$ $V_{e}(x) w_{e}=0$ in the whole domain $B$ with $w_{e}=0$ on $\partial B$, multiplying by $w_{e}^{+} \chi_{B(e)}+$ $w_{e}^{-} \chi_{B(-e)}$ and integrating over $B$ we obtain

$$
\begin{aligned}
0= & \int_{B} \nabla w_{e} \nabla\left(w_{e}^{+} \chi_{B(e)}+w_{e}^{-} \chi_{B(-e)}\right) d x-\int_{B} V_{e}(x) w_{e}\left(w_{e}^{+} \chi_{B(e)}+w_{e}^{-} \chi_{B(-e)}\right) d x \\
= & \int_{B}\left(\left|\nabla\left(w_{e}^{+} \chi_{B(e)}\right)\right|^{2}+\left|\nabla\left(w_{e}^{-} \chi_{B(-e)}\right)\right|^{2}\right) d x \\
& -\int_{B} V_{e}(x)\left[\left(w_{e}^{+} \chi_{B(e)}\right)^{2}+\left(w_{e}^{-} \chi_{B(e)}\right)^{2}\right] d x \\
= & \int_{B}\left|\nabla w_{e}^{1}\right|^{2} d x-\int_{B} V_{e}(x)\left(w_{e}^{1}\right)^{2} d x .
\end{aligned}
$$


Now we can use the comparison between the potential $V_{e}(x)$ and $V_{e s}(x)$, given by (3.4), and obtain from (3.12)

$$
0 \geq \int_{B}\left|\nabla w_{e}^{1}\right|^{2} d x-\int_{B} V_{e s}(x)\left(w_{e}^{1}\right)^{2} d x=Q_{e s}\left(w_{e}^{1}, w_{e}^{1}\right)=Q_{u}\left(w_{e}^{1}, w_{e}^{1}\right),
$$

because $w_{e}^{1}$ is a symmetric function with respect to $\sigma_{e}$. Similarly we can show

$$
Q_{u}\left(w_{e}^{2}, w_{e}^{2}\right) \leq 0
$$

Now, for every $e \in S^{*}$, we let $\psi_{e} \in \mathcal{V}_{0} \cap H_{0}^{1}(B)$ be defined by

$$
\psi_{e}(x)=\left(\frac{\left\langle w_{e}^{2}, \varphi_{1}\right\rangle}{\left\langle w_{e}^{1}, \varphi_{1}\right\rangle}\right)^{1 / 2} w_{e}^{1}(x)-\left(\frac{\left\langle w_{e}^{1}, \varphi_{1}\right\rangle}{\left\langle w_{e}^{2}, \varphi_{1}\right\rangle}\right)^{1 / 2} w_{e}^{2}(x) .
$$

Using (3.11), it is easy to see that $e \mapsto \psi_{e}$ is an odd and continuous map from $S^{*}$ to $\mathcal{V}_{0}$. By construction, $\left\langle\psi_{e}, \varphi_{1}\right\rangle=0$ for all $e \in S^{*}$. Moreover, since $w_{e}^{1}$ and $w_{e}^{2}$ have disjoint supports, (3.13) and (3.14) imply

$$
Q_{u}\left(\psi_{e}, \psi_{e}\right) \leq 0 \quad \text { for all } e \in S^{*} .
$$

Recalling (3.9), we now distinguish the following cases:

Case 1: $\mu_{0} \geq 2$. Then let $\lambda_{1}, \tilde{\lambda}_{2}, \ldots, \tilde{\lambda}_{\mu_{0}}$ be the negative eigenvalues of the operator $L_{0}$ in increasing order, and let $\varphi_{1}, \tilde{\varphi}_{2}, \ldots, \tilde{\varphi}_{\mu_{0}} \in \mathcal{V}_{0}$ be corresponding $L^{2}$-orthonormal eigenfunctions. Similarly as in (3.7) we have

$$
\inf _{\substack{v \in H_{0}^{1}(B) \cap \nu_{0}, v \neq 0 \\\left\langle v, \varphi_{1}\right\rangle=\left\langle v, \tilde{\varphi}_{2}\right\rangle=\ldots=\left\langle v, \tilde{\varphi}_{\mu_{0}}\right\rangle=0}} \frac{Q_{u}(v, v)}{\langle v, v\rangle} \geq 0 .
$$

We now consider the map $h: S^{*} \rightarrow \mathbb{R}^{\mu_{0}-1}$ defined by $h(e)=\left[\left\langle\psi_{e}, \tilde{\varphi}_{2}\right\rangle, \ldots,\left\langle\psi_{e}, \tilde{\varphi}_{\mu_{0}}\right\rangle\right]$. Since $h$ is an odd and continuous map defined on a $N-k-1$-dimensional sphere and $\mu_{0} \leq N-k, h$ must have a zero by the Borsuk-Ulam Theorem. Hence there is $e \in S$ such that $\left\langle\psi_{e}, \tilde{\varphi}_{k}\right\rangle=0$ for $k=2, \ldots, \mu_{0}$ and, by construction, $\left\langle\psi_{e}, \varphi_{1}\right\rangle=0$. Since $\psi_{e} \in \mathcal{V}_{0}$ and $Q_{u}\left(\psi_{e}, \psi_{e}\right) \leq 0$, the function $\psi_{e}$ is a mimimizer for the quotient in (3.16). Consequently, it must be an eigenfunction of the operator $L_{0}$ corresponding to the eigenvalue zero. Hence $\psi_{e} \in C^{2}(\bar{B})$, and $\psi_{e}$ solves $-\Delta \psi_{e}-V_{u}(x) \psi_{e}=0$ in $B$. Moreover, $\psi_{e}=0$ on $H(e)$ by the definition of $\psi_{e}$, and $\partial_{e} \psi_{e}=0$ on $H(e)$, since $\psi_{e}$ is symmetric with respect to the reflection at $H(e)$. From this it is easy to deduce that the function $\hat{\psi}_{e}$ defined by

$$
\hat{\psi}_{e}(x)= \begin{cases}\psi_{e}(x), & x \in B(e), \\ 0, & x \in B(-e),\end{cases}
$$

is also a (weak) solution of $-\Delta \hat{\psi}_{e}-V_{u}(x) \hat{\psi}_{e}=0$. This however contradicts the unique continuation theorem for this equation (see e.g. [12, p. 519]).

Case 2: $\mu_{0}=1$. Then, for any $e \in S^{*}$, since $\psi_{e} \in \mathcal{V}_{0},\left\langle\psi_{e}, \varphi_{1}\right\rangle=0$ and $Q_{u}\left(\psi_{e}, \psi_{e}\right) \leq 0$, the function $\psi_{e}$ must be a Dirichlet eigenfunction of the operator $L_{0}$ corresponding to the eigenvalue zero. This leads to a contradiction as in Case 1.

Since in both cases we reached a contradiction, there must be a direction $e \in S^{*}$ such that $w_{e}$ does not change sign on $B(e)$. Hence (3.10) holds either for $e$ or for $-e$. Now, to finish the proof, we again distinguish two cases. Suppose first that $w_{e} \equiv 0$ on $B$. Since $S^{*} \cap S_{*}=\varnothing$, we then have $\lambda_{1}\left(e, V_{u}\right) \geq 0$, and thus Proposition 2.3 yields the foliated Schwarz symmetry of $u$. Suppose next that $w_{e} \not \equiv 0$, which implies 
that $w_{e}>0$ in $B(e)$ by $(3.10)$ and the strong maximum principle, noting that $w_{e}$ solves (3.2). Then also $\lambda_{1}\left(e, V_{e}\right)=0$, where as before $\lambda_{1}\left(e, V_{e}\right)=0$ denotes the first Dirichlet eigenvalue of $-\Delta-V_{e}(x)$ on $B(e)$. To get a direction $e^{\prime}$ such that $H\left(e^{\prime}\right)$ is a symmetry hyperplane for $u$ and $\lambda_{1}\left(e^{\prime}, V_{u}\right)=\lambda_{1}\left(e^{\prime}, V_{e^{\prime}}\right)=0$, we argue exactly as in the proof of Proposition 3.1. Hence, starting from (3.10) we rotate the hyperplane until we reach a hyperplane $H\left(e^{\prime}\right)$ corresponding to a unit vector $e^{\prime}$ for which $w_{e^{\prime}} \equiv 0$. Again the assertion now follows from Proposition 2.3.

If $S_{*}=\varnothing$ we repeat all the steps starting from (3.10), taking $S^{*}=S$ and substituting $H_{0}^{1}(B) \cap \mathcal{V}_{0}$ with $H_{0}^{1}(B)$. Again we reach a direction $e^{\prime}$ (possibly equal to $e)$ such that $w_{e^{\prime}} \equiv 0$ and thus $\lambda_{1}\left(e^{\prime}, V_{u}\right) \geq 0$ because $S_{*}=\varnothing$. The proof is finished.

Proof of Theorem 1.2, Let $u$ be a solution with Morse index $m(u) \leq N$. In the proof of Theorem 1.1 we have obtained a symmetry hyperplane $H(e)$ for $u$ such that $\lambda_{1}\left(e, V_{u}\right) \geq 0$. On the other hand, if the nodal set does not intersect the boundary, the argument of [1], which works regardless the sign of $f(0)$, shows that $\lambda_{1}\left(e, V_{u}\right)<0$. Hence the assertion holds.

\section{REFERENCES}

[1] Aftalion, A. and Pacella, F., Qualitative properties of nodal solutions of semilinear elliptic equations in radially symmetric domains, C. R. Math. Acad. Sci. Paris 339 (2004), 339-344. MR2092460 (2005f:35086)

[2] Bartsch, T. and Weth, T., A note on additional properties of sign changing solutions to superlinear elliptic equations, Topol. Methods Nonlinear Anal. 22 (2003), 1-14. MR2037264 (2005d:35056)

[3] Bartsch, T., Weth, T. and Willem M., Partial symmetry of least energy nodal solutions to some variational problems, J. Anal. Math. 96 (2005), 1-18. MR2177179

[4] Berestycki, H. and Nirenberg, L., On the method of moving planes and the sliding method, Bol. Soc. Bras. Mat., 22 (1991), 1-37. MR1159383 (93a:35048)

[5] Brock, F., Symmetry and monotonicity of solutions to some variational problems in cylinders and annuli. Electron. J. Diff. Eq. 2003, 1-20. MR2011581 (2004k:49086)

[6] Castorina, D. and Pacella, F., Symmetry of positive solutions of an almost-critical problem in an annulus, Calc. Var. P.D.E. 23 (2005), 125-138. MR2138079 (2006a:35109)

[7] Castorina, D. and Pacella, F., Symmetry of solutions of a semilinear elliptic problem in an annulus, Progress in Nonlinear Diff. Eq. and Appl. 66 (2005), 135-148. MR2187799

[8] de Figueiredo, D.G. and Gossez, J.-P., Strict monotonicity of eigenvalues and unique contination, Comm. Partial Differential Equations 17 (1992), 339-346. MR.1151266 (93b:35098)

[9] Gidas, B., Ni, W.-M. and Nirenberg, L., Symmetry and related properties via the maximum principle, Comm. Math. Phys. 68 (1979), 209-243. MR0544879 (80h:35043)

[10] Pacella, F., Symmetry results for solutions of semilinear elliptic equations with convex nonlinearities, J. Funct. Anal. 192 (2002), 271-282. MR1918496 (2003m:35082)

[11] Serrin, J., A symmetry problem in potential theory, Arch. Ration. Mech. 43 (1971), 304-318. MR.0333220 (48:11545)

[12] Simon, B., Schrödinger Semigroups, Bull. Am. Math. Soc. New Ser. 7 (1982), 447-526. MR0670130 (86b:81001a)

[13] Smets, D. and Willem, M., Partial symmetry and asymptotic behaviour for some elliptic variational problems, Calc. Var. Part. Diff. Eq. 18 (2003), 57-75. MR2001882 (2004m:35092)

Dipartimento di Matematica, Universitá di Roma "La Sapienza", P. le A. Moro 2, 00185 Roma, Italy

Mathematisches Institut, Universität Giessen, Arndtstr. 2, 35392 Giessen, Germany 